\title{
REFRESCO Y DIABETES ENTRE LOS MAYAS DE TENEJAPA, San Cristóbal de Las Casas y Chamula, Chiapas
}

\author{
Soft Drinks and Diabetes among the Maya of Tenejapa, \\ San Cristóbal de Las Casas, and Chamula, Chiapas \\ Jaime Tomás Page-Pliego
}

Resumen: Este trabajo aborda diferentes factores asociados a la importancia que ha adquirido el consumo de refresco, específicamente de Coca-Cola, entre los mayas que habitan en las cabeceras municipales de Tenejapa, Chamula y San Cristóbal de Las Casas, Chiapas, en la génesis y agravamiento de la obesidad y la diabetes. Los datos de campo arrojan cómo la situación de un número considerable de diabéticos y sus familiares sin antecedentes previos de diabetes se vincula a un intenso consumo de refresco previo a la aparición de la sintomatología. El trabajo, además de revisar las representaciones que los mayas van construyendo en torno a dicha problemática, muestra pormenores de diferente orden que facilitan la promoción y venta de refresco en la región.

Palabras clave: refresqueras, diabetes, representaciones sociales.

Abstract: This paper deals with various factors associated with the importance attached to consumption of soft drinks, specifically CocaCola among the Maya living in the municipalities of Tenejapa, Chamula and San Cristobal de Las Casas, Chiapas as cause and exacerbation of obesity and diabetes. The field data show a clear link between a significant number of diabetic sand their relatives with no previous history of diabetes, linked to heavy soda consumption before the onset of symptoms. The work in addition to reviewing the representations that the Maya before us are building around this problem, shows details of different order that facilitate the promotion and sale of soda in the region.

Keywords: soft drink industries, diabetes, social representations.

Jaime Tomás Page-Pliego, doctor en Antropología Social por la Facultad de Filosofía y Letras de la UNAM. Investigador en PROIMMSE-IIA-UNAM. Temas de especialización: enfermedades del rezago y emergentes, etnomedicina. Correo electrónico: jaimepagepliego@comunidad.unam.mx.
Enviado a dictamen: 30 de mayo de 2012 Aprobación: 22 de agosto de 2012 Revisiones: 1 


\section{Introducción}

$\mathrm{U}$ n problema relacionado con la epidemia de diabetes en México es el ilimitado e irrestricto consumo de refrescos - bebidas carbonatadas y azucaradas-. Al respecto se ha documentado la importancia que este tipo de bebidas tiene en la génesis e incremento de los índices de sobrepeso, obesidad, diabetes mellitus y gota (Committee on School Health, 2004; Ludwig et al., 2001; U.S. Department of Health and Human Services, 2001; Organización Mundial de la Salud, 2003; Schulze et al., 2004; Choi et al., 2008; Ludwig et al., 2001; citados en Jordan, 2008: 149-150).

Bajo la consideración de que los condicionantes y determinantes de la epidemia de diabetes presente en Los Altos de Chiapas son diversos, este trabajo tiene como objetivo presentar una reflexión sobre las condiciones que han coadyuvado, por parte de la industria refresquera, específicamente de la empresa Coca-Cola FEMSA, a la génesis y prevalencia de la diabetes mellitus en las cabeceras municipales de Tenejapa, Chamula y San Cristóbal de Las Casas, Chiapas, matizada con las opiniones que los enfermos han expresado en torno al tema. Los datos provienen de información preliminar de la investigación denominada "Representaciones sociales y estrategias de atención en torno a diabetes mellitus en población de la periferia de San Cristóbal de Las Casas y cabeceras municipales de Chamula y Tenejapa, Chiapas."

Los "actores significativos" considerados en torno a esta problemática son los sujetos de la investigación, es decir, diabéticos tsotsiles y tseltales que no recurren -o lo hacen irregularmente- a los servicios de salud, y la empresa refresquera Coca-Cola FEMSA. Por otro lado, los "actores secundarios" son los grupos de poder de los municipios - los cuales inciden en las prácticas sociales de orden cívico-religioso-, el subsistema de salud dirigido a población excluida y elementos de organización familiar y social.
En lo concerniente a los "actores sujetos", se realizaron 51 entrevistas a diabéticos, de los cuales 47 son mayas y 4 mestizos; de estas entrevistas, 22 se realizaron en Chamula, 12 en San Cristóbal de Las Casas y 17 en Tenejapa. Se documentaron representaciones sobre diabetes mellitus y sobre las causas a las que atribuyen el haber enfermado, así como sobre el devenir pormenorizado de la trayectoria desde que se asumieron como diabéticos. Para este documento se han entresacado opiniones y experiencias en torno al consumo de refresco y su repercusión en la génesis de la diabetes y su agravamiento.

Por lo que respecta al actor empresarial, nos hemos basado en la revisión documental relativa a la refresquera, principalmente en dos documentos que abordan el problema, tomando como eje Los Altos de Chiapas (Jordan, 2008; Blanding, 2010) e información que circula en las redes sobre la empresa Coca-Cola; asimismo, nos basamos en datos etnográficos propios relativos a las estrategias de mercadotecnia y ventas.

En lo relacionado con actores secundarios y otros elementos, los datos provienen de resultados de otras investigaciones propias, así como de datos obtenidos durante el curso de la investigación que cobija este trabajo.

¿Por qué refresqueras y diabetes mellitus? Entre otros temas, corresponde a la antropología médica desentrañar y analizar los diferentes factores e interrelaciones que determinan las condiciones de salud, la génesis e incremento de los índices de morbimortalidad, y las formas que adquiere la atención en los diferentes conjuntos sociales, dando mayor peso a aquellos factores en los que resulta imprescindible intervenir para coadyuvar a su prevención o evitar su agravamiento y complicaciones. Al respecto, no es posible soslayar la estrecha relación entre el consumo persistente de refrescos característico de la región de estudio, y la variación en el tiempo de los índices de glucosa en sangre, con el correspondiente incremento de la producción de insulina en páncreas, el eventual 
agotamiento de la trama celular que produce la hormona $\mathrm{y}$, concomitantemente, el incremento de los índices de obesidad y diabetes mellitus.

Los aspectos teóricos y metodológicos se ciñen a los del proyecto que lo sustenta. En este sentido, siguiendo a Menéndez (2002: 336), se ha organizado la indagatoria desde el enfoque relacional, que sitúa al objeto de estudio en el contexto más amplio que lo condiciona/determina; se ocupa particularmente "de las condiciones y significaciones de subalternidad", y agregaría subordinación, inmersas en relaciones de hegemonía-dominación y marginación (Menéndez 2002: 135). En relación con nuestro tema, dicha propuesta permite dar cuenta de las simetrías/ asimetrías existentes en torno a la diabetes mellitus, toda vez que los sujetos que han participado en la investigación pertenecen a conjuntos sociales históricamente colonizados, se ubican en contextos en los que predomina la pobreza extrema, así como la exclusión social y económica y, por todo lo anterior, sufren rezagos importantes en materia educativa, de salud, servicios sanitarios, vivienda y disposición de alimentos. Con respecto al último rubro, y de interés para este trabajo, predominan perfiles dietéticos en los que sobresalen dietas hipercalóricas e hipoproteicas que son resultado, entre otros factores, del efecto causado por la desinformación mediática que estimula el consumo de alimentos hipercalóricos, problemática que en la región se agudiza por el impacto que están teniendo en el consumo las estrategias de mercado de las industrias refresqueras y de embolsados salados $\mathrm{y}$ dulces.

El enfoque relacional, a su vez, torna pertinente el concepto de vulnerabilidad en salud, tal y como propone Paul Farmer (2001), donde aspectos como la clase, la etnia y el género interactúan dinámicamente con las inequidades sociales y económicas en la configuración de escenarios particulares de vulnerabilidad.

Elpunto de vista de los diferentes actores que inciden de manera importante en el devenir de la problemática considerada —el enfermo, el operador médico, la institución biomédica, los medios de comunicación o las empresas - constituye el eje metodológico a partir del cual se organiza la investigación. Entre estos actores destacan, para este trabajo, el enfermo y la empresa refresquera.

\section{Coca-Cola en Los Altos de Chiapas}

Como veremos, para el tema que nos ocupa Los Altos de Chiapas se caracterizan porque el consumo del refresco Coca-Cola es el más elevado del mundo, eso sin considerar el alto consumo de otras gaseosas azucaradas, entre las que destacan Pepsi, Fanta, Fresca $y$, en menor medida, otras marcas de capital mexicano como Fersán.

$\mathrm{Al}$ respecto, un estudio en el que se revisaron las ventas mundiales de The Coca-Cola Company (TCCC) resalta que, mientras en la mayor parte de los países del orbe, considerando el total de población de los mismos, en promedio se consumen cien tazas de 250 mililitros por persona al año, en los Estados Unidos se consumen cuatrocientas y en México seiscientas (Blanding, 2010: 290). Este dato sitúa a México como el principal consumidor de esta bebida en el mundo. Sin duda fue éste el motivo por el cual, durante el Foro económico que este año 2012 tuvo lugar en Davos, Suiza, Muhtar Kent, presidente internacional de TCCC, franqueado por Felipe Calderón, anunció que invertiría cinco mil millones de dólares en México en un lapso de cinco años a partir de 2012 (Guthrie, 2012; EFE, 2012).

El promedio de consumo de Coca-Cola señalado para México no necesariamente refleja las variaciones de consumo presentes en las diferentes regiones del país. En este sentido, por ejemplo, mientras que en Los Altos de Chiapas - con un aproximado de 928073 habitantes registrados en el censo de 2010 que incluye mayas y no indígenas (INEGI, 2010) - se consumen en promedio 3285 tazas por persona al año, lo que en litros equivale a 821.25, es decir, 2.25 litros al día.Por poner 
otro ejemplo, entre la población comcáac del caluroso desierto de Sonora — que en el censo de 2010 arrojó un total de 807 habitantes (Yáñez, 2012: 105)—, se llegan a consumir en tiempos de calor hasta cinco mil tazas del oscuro líquido por año, lo que puesto en litros varía a lo largo del año entre dos y cinco por persona por día (Yáñez, 2012: 141).

Si el consumo de refresco se traduce a kilocalorías, según se señala en Pérez et al. (2012: 83), el refresco de Cola de 355 mililitros contiene 152 kilocalorías por tanto, una porción de 500 mililitros, que es la cantidad que contiene el envase de vidrio que se comercializa en las comunidades mayas de Los Altos de Chiapas, tendrá un aproximado de 214 calorías, por lo que al final del día, según el consumo señalado en el párrafo anterior, entre cuatro y cinco refrescos aportarían entre 856 y 1070 kilocalorías, considerando que los requerimientos kilocalóricos por día rondan entre 1500 y 2000. A eso, por supuesto, tenemos que agregar las calorías que aporta una tortilla de 60 gramos de maíz de nixtamal hecha en casa, lo que da un aproximado de 106 kilocalorías (una tortilla de 30 gramos aporta 53 kilocalorías (Pérez, 2012: 37). Es inevitable considerar este alimento porque constituye la base de la alimentación del campesinado del México mesoamericano, distinguiéndose por ello de la población del norte de México, donde se prefiere la tortilla a base de harina de trigo. El promedio de consumo de tortillas al día entre los mayas de Los Altos que nos ocupan es de entre 15 y 20 piezas por adulto, lo que resulta en un rango de entre 1590 y 2120 kilocalorías.

Si se suman las calorías proporcionadas por la tortilla y por el refresco, nos arroja un aporte calórico que varía entre 2246 y 3190 kilocalorías al día, lo que rebasa en mucho las 1500 kilocalorías que el cuerpo requiere. Ello sin tomar en cuenta las calorías aportadas por otros alimentos como el frijol, las verduras, la carne de pollo y la carne roja, además de alimentos fritos, dulces y chocolates. El panorama que arrojan estos datos resulta aterrador.

\section{Producción de Coca-Cola en San Cristóbal}

Dos circunstancias favorecen enormemente el fácil acceso por parte de la población a los productos de ofrece la embotelladora Coca-Cola FEMSA. Por un lado, que en la ciudad de San Cristóbal de Las Casas, eje político, administrativo y económico de Los Altos de Chiapas, se asienta una de las dos plantas más grandes que dicha empresa posee en México, propiedad de un ramal denominado Inmuebles del Golfo S.A. de C.V. (ver foto 1). La otra se encuentra en Apizaco, Tlaxcala. La planta ubicada en San Cristóbal, propiedad de la corporación embotelladora FEMSA - que antes fuera grupo Monterrey-, se sitúa en las faldas del antiguo volcán conocido como Huitepec, que alcanza una elevación aproximada de 2700 metros sobre el nivel del mar - cuya cúspide se alcanza a ver en el extremo izquierdo de la foto 2-. Uno de los calificativos que los lugareños dan a este promontorio es el de "volcán de agua".

Con el agua que en 2008 se extrajo del pozo profundo que la empresa perforó a las faldas del Huitepec, se produjeron 120000 litros de refresco al día, lo que dio un total de 43800000 litros en dicho año. Esta cantidad hubiera sido suficiente para surtir Chiapas, parte de Oaxaca y Tabasco (Jordan, 2008: 75). No considerando eso suficiente, en el año 2012 se está cavando un segundo pozo.

El agua que la empresa FEMSA extrae del Huitepec constituye un hito de reclamo por parte de la población y de la administración de la ciudad. Sin embargo, durante las gestiones de Ernesto Zedillo y Vicente Fox varios exfuncionarios de Coca-Cola FEMSA formaron parte de sus gabinetes, como Luis Téllez Kuenzler, miembro del comité administrativo de FEMSA, quien fuera secretario de energía durante la administración de Zedillo. Por otro lado, Vicente Fox, quien sucedió a Zedillo en la silla presidencial, fue gerente general de dicha empresa y, durante su gestión, además de Tellez, quien fungiera como secretario de transporte en dicho sexenio, 
también fueron parte del gabinete: Reyes Tamez Guerra, secretario de educación; Fernando Canales Clariond, en diferentes momentos secretario de Economía y Energía; Fernando Elizondo Barragán, secretario de Energía; y María Teresa Herrera Tello, secretaria de Reforma Agraria. (Castro Soto, 2003-5; FEMSA, 2006b; Rodríguez Gómez, 2008; Campos et al., 2008, citados en Jordan, 2008: 70). Uno de los nombramientos de mayor relevancia para el tema que nos ocupa fue el de Cristóbal Jaime Jáquez como comisionado nacional del agua, quien antes fuera director general de Coca-Cola México. Durante su gestión se otorgó una concesión de 20 años a dicha empresa para la extracción de agua sin costo alguno, incluyendo exoneraciones en el pago de impuestos y otras cuotas, con la salvedad de una cantidad simbólica que recibe el Gobierno federal, no el estatal ni el local de la ciudad de San Cristóbal (Blanding, 2010: 167).

\section{La llegada de la Coca-Cola}

Un elemento de suma importancia para la penetración de la empresa Coca-Cola en Los Altos de Chiapas fue la concesión de la distribución de refresco a diferentes grupos de poder de los municipios aledaños a San Cristóbal de Las Casas. Este hecho tuvo lugar después del empuje que a partir de 1939 dio el entonces denominado Departamento de Acción Social, Cultural y de Protección al Indígena (DASCPI) (Favre, 1973: 74) para colocar a jóvenes mayas con cierto grado de escolaridad como secretarios de cabildo en sustitución del personal ladino que detentaba en provecho propio dichos puestos. Al situar a dichos jóvenes en la estructura de gobierno civil municipal, se abrió la puerta para el gradual control de los gobiernos municipales e instancias religiosas y, al cabo, para la conformación de poderosos cacicazgos.

In many communities, the same caciques that monopolized production of pox retained the concessions to coke and later Pepsi. In some, such as
Amatenango, concessions were granted politically, with officials of the Institutional Revolutionary Party (PRI) controlling Coke and the Party of the Democratic Revolution (PRD) controlling Pepsi. It was easy enough to substitute the new drinks for many of the same rituals that previously used pox (though in some cases, such as the church in Chamula, pox is still maintained in limited quantities). Those owning the concessions of the soft drinks became rich, reaping huge profits in villages with little other commerce or industry, and passing the concessions along to family members to create dynasties. Before long, however, the increasing consumption of soft drinks brought its own problems-tooth decay, diabetes, and obesity (Blanding, 2010: 157-158).

Uno de los casos mejor documentados sobre este fenómeno es el del grupo de los Tuxumetik de Chamula, apelativo que adquirieron Salvador López Tuxum, quien lo liderara, secundado por Juan Gómez Oso y Salvador Sánchez Gómez (Robledo 1987: 69-70). López Tuxum:

[...] aseguró ser el único intermediario tanto para asuntos de orden sociopolítico como económico con el mundo ladino. Su visión comercial, poco a poco llevó al grupo a constituirse en el principal acaparador de la producción del municipio, ser el distribuidor de los productos con mayor demanda (Coca-Cola, Pepsi y cerveza), establecerse como usurero y constituirse prácticamente como dueño de la mayor parte de la cooperativa de transportes que fue fundada con apoyo del Instituto Nacional Indigenista, previas gestiones del grupo ante la institución (Page, 2005: 48-49).

En un testimonio que recabó Jordan del hijo de López Tuxum, Carlos López Gómez, se señala:

Todo [este negocio] es herencia de mi finado padre. [...] Mi padre [Salvador López Tuxum] es el que 
obtuvo primero la concesión, el permiso pues, del distribuidor. [...] Nos platica mi mamá que [antes] no se conocía ni la Coca ni la Pepsi. Se conocía otro tipo de producto que se llamaba gaseosa.

Pero los empresarios de las refresqueras Coca y Pepsi vieron que sí se vendía el producto de gaseosa, [...] y vinieron [en 1962] a ofrecer [la concesión]. Entonces [fue allí] donde se empezó a introducir el producto Coca y Pepsi. [El refresco rápidamente] obtuvo más demanda, más ventas. Entonces el producto anterior, la gaseosa, ya no se vendía tanto. Lo que se empezó a vender más es la Coca y la Pepsi.

Entonces mi papá llegaba a San Cristóbal a acarrear producto, pero no en vehículo, sino en caballo. No había carretera ni medio de trasladarse con carros, entonces llegaba a acarrear allí. [...] Y la gente venía acá [al depósito en la cabecera San Juan Chamula], en este mismo lugar, a comprar [desde] las diferentes comunidades. Venían acá a acarrear con mecate o con caballo. Venían a traer acá porque mi papá tampoco tenía carro para distribuir, por eso venían los paisanos a comprar. [...] Por lo regular empezó poco a poco. Sólo los más cercanos de la cabecera venían a [a] carrear. Ya en los parajes o las colonias más retirados, no muy conocían. Lo más lejos que [caminaban eran] unos 30-40 kilómetros (López Gómez, 2007, en Jordan, 2008: 74).

El prestigio social que dio en Chamula ofrecer CocaCola y Pepsi, principalmente la primera, en festejos familiares y fiestas patronales, así como en juegos de basquetbol, rápidamente se extendió por todos Los Altos de Chiapas.

\section{Estrategias de expansión del mercado y monopolización}

Las estrategias para la expansión del mercado entre la población alteña no se diferencian en gran medida de las empleadas en cualquier otro contexto mundial; pueden resumirse, dado que éste no es el ámbito para un tratamiento a profundidad del tema, en la presión que ejercen los agentes de ventas de las empresas en las zonas rurales con objeto de impulsar un mayor consumo mediante diversas acciones: ofrecer refrigeradores, que tienen que ser únicamente destinados a los productos de la propia marca; pintar los locales con el logo de las empresas; ofrecer toldos, mesas y sillas también con dicho logo; instalar espectaculares en lugares estratégicos de tal manera que pueden ser observados desde distancias considerables y por un número elevado de gente; o pintar las canchas de basquetbol con los colores y el logo de la empresa.

Otra estrategia consiste en exigir a los comerciantes exclusividad para la venta del producto, como señala el testimonio de López Gómez:

Al fallecer mi padre, se seguían vendiendo los dos productos, las dos marcas. Tenemos camioncitos para distribuir en los parajes, y en esos días se llevaba Coca y Pepsi. Pero como le decimos, tanto de la Pepsi como la Coca nos presionaban en ambas partes. "Vendes más Coca, te apoyamos. Y no quiero que mezclen con otro producto," decían los de la Coca. [...] Y ya después, nos obligaron definitivamente: "Tú decides: te quedas con la Coca o te vas con la Pepsi”. Así sucesivamente seguían presionando. Nosotros nos dimos cuenta que ya no podíamos seguir trabajando las dos marcas. iPor quée Por la misma razón: te obligaban. O te decían “¿Cuánto quieres pa' que se pierda la Pepsi?" Quiere que quedemos como exclusivo de Coca. [...] Por esa razón nosotros ya decidimos quedarnos con la marca de Coca-Cola (López, 2007, en Jordan, 2008: 77).

En cuanto a estimular el consumo familiar, como ya se señaló, se agrega el hecho de que los refrescos de Coca-Cola se venden más baratos en las comunidades de Los Altos, estrategia que la empresa ha denominado "brindar precios accesibles" (Javier, 2008, en Jordan, 2008: 76). 
La dimensión que ha alcanzado el refresco como elemento imprescindible en la dieta de los mayas que nos ocupan, así como su consumo en los diferentes eventos sociales y religiosos que tienen lugar, lo resume adecuadamente Jordan. El refresco se ingiere:

- en vez de agua o pozol como alimento básico;

- en la compañía de otros como una demostración de amistad o respeto;

- $\quad$ para pedir o agradecer un favor;

- al momento de pagar una deuda;

- mezclada con pox para distraerse u olvidarse de cosas negativas;

- como sustituto para pox o vino en ceremonias y ofrendas católicas sincréticas;

- como parte de los rituales de curación, etcétera (Jordan, 2008: 145).

Cuando nace un niño, hay boda o velorio, se ofrece refresco. La cantidad que se ofrezca dependerá del evento y de la situación económica de la familia, y puede ser de tres o cuatro cajas o hasta de cien. Por ejemplo, para un mitin de campaña, un candidato compró cinco tráileres de Coca-Cola, cada uno con 180 cajas, totalizando más de 20000 botellas (Blanding, 2010: 158-159).

Un estudio realizado por COMPITCH tanto en Los Altos de Chiapas como en la Selva concluyó que la mayor parte de los casos de diabetes se presentan en las comunidades que están a pie de carretera, siendo menor la incidencia donde se dificulta la llegada del refresco (Blanding, 2010: 159-160).

Al respecto, una de las personas entrevistadas en el proyecto que cobija este trabajo señalaba que antes de que llegará la carretera no había "gordura, azúcar o la presión alta":

Cuando ya llegó la carretera, con la carretera ya llegó todo. [...] Aproximadamente, ahorita te digo, como de año de 70 por ahí, no exactamente pero por ahí.
Porque antes llegaba muy poco porque tenías que traer cargando hasta acá desde San Cristóbal, pero cuando ya llegaron los camiones hubo producto hasta en la ranchería (entrevista Tenejapa AN 22 de septiembre de 2010).

Al transitar por los caminos y carreteras de Los Altos de Chiapas, es inevitable toparse a cada paso con la publicidad de Coca-Cola y de otras refresqueras, ya en casas y comercios o bien en letreros y espectaculares donde se da la bienvenida a la comunidad (ver fotos 3, 4 y 5). Asimismo, al asomarse a las casas que están al paso, invariablemente pueden observarse pilas de cajas con envases de Coca-Cola, algunas llenas, o bien, dependiendo del día, cajas de refresco vacías colocadas a pie de carretera en espera del paso del camión. Al deambular por las calles invariablemente se topa uno con adultos, adolescentes y niños platicando con una botella del oscuro líquido en las manos o en el piso a un lado de su dueño.

Estos elementos destacan aún más cuando en las historias de los diabéticos resaltan, como factores clave para el desencadenamiento de la diabetes y la obesidad, la importancia que se atribuye a estas bebidas y los cambios significativos que ha tenido la dieta en las últimas décadas.

\section{La influencia proselitista}

Considero que un factor que ha coadyuvado al alto consumo de refresco puede atribuirse al embate proselitista que diferentes grupos de cristianos de filiación calvinista han llevado a cabo en el estado. La bandera que han esgrimido en lo tocante a nuestro tema refiere a la satanización de prácticas religiosas tradicionales y, en consecuencia, a la insidiosa campaña en contra del uso de aguardiente de caña (pox). Al respecto, sobre la actualización de conocimiento y cosmovisión que por la vía de la revelación onírica recibió un prestigiado j-ilol de Chenalhó, éste señaló: 
Primero, te entregaron el pox, pero ahora te quiero decir que no lo debes de beber, así que te lo vine a quitar. El trago no es nuestro, es del enemigo, y te lo digo para que lo sepas y lo tengas en tu mente (Page, 2005: 351).

Por este motivo, el pox desde entonces se destina a las deidades del mal y pasa a ocupar su lugar el refresco — su olor dulzón - como nutrimento de las deidades celestiales o del bien.

\section{Obesidad y diabetes en Chiapas}

La Organización para la Cooperación y el Desarrollo Económicos (OCDE) informó que México ocupó en 2010 el primer lugar de obesidad en el mundo. En dicho año, el 30\% de los mexicanos era obeso, mientras que el 70\% tenía sobrepeso (OCDE, 2010).

En lo que concierne al estado de Chiapas, según señala la Encuesta Nacional de Salud y Nutrición 2006, $16.7 \%$ de la población entre cinco y 11 años sufría de sobrepeso y obesidad, registrándose a nivel urbano $25.8 \%$, y a nivel rural $10.3 \%$.

Respecto a los adolescentes, se registró que 26.9\% sufría sobrepeso y obesidad, contabilizándose $29.9 \%$ a nivel urbano y $24.6 \%$ en el ámbito rural.

Entre los adultos, $63.7 \%$ sufría sobrepeso y obesidad. De ese porcentaje, $66.5 \%$ estaba afectado de obesidad abdominal. Si a su vez se diferencian los porcentajes entre las zonas rurales y las urbanas, se registraron respectivamente porcentajes de $68 \%$ y $71.6 \%$ en relación con el sobrepeso, y $59 \%$ y $60.8 \%$ por lo que respecta a obesidad (ENSANUT, 2007).

Es evidente que los niveles de sobrepeso y obesidad en los tres grupos, así como la obesidad central entre los adultos, rebasó la media nacional en 2006. En 2012 todavía no se cuenta con información, pero lo esperado es que dichos índices hayan aumentado sensiblemente.

Respecto a la obesidad y su causalidad, a su vez, la ENSANUT 2006 (2007) señala:
La obesidad es un grave problema de salud pública, resultante de la ingestión de dietas elevadas en alimentos con alta densidad energética, bajos en fibra, y al elevado consumo de bebidas con aporte energético, en combinación con una escasa actividad física. Esta última se ha asociado a la urbanización, al crecimiento económico, a los cambios en la tecnología para la producción de bienes y servicios, así como a los actuales estilos de recreación (ENSANUT, 2007: 76).

En este sentido, el Gobierno mexicano reconoce que uno de los grandes problemas nacionales es "el consumo elevado de bebidas con aporte energético", por no decir refrescos. Sin embargo, a pesar de que en el estado seis de cada diez adultos presenta sobrepeso y obesidad, problema no menor en el país, ni en la ENSANUT 2006 (2007) ni en ningún otro documento oficial aparece dato alguno que apunte hacia una política encaminada a limitar el uso de refrescos, ni siquiera, por ejemplo, leyendas en los envases que indiquen el riesgo que implica su consumo excesivo.

Por lo que respecta a los diabéticos, la ENSANUT 2006 reportó que solamente se detectaron 369.1 casos entre las personas que acudieron a solicitar atención. Estos datos resultan un tanto engañosos para la consideración epidemiológica ya que, al registrar las instituciones de salud solamente la demanda de atención, dejan fuera de los registros a la mayor parte de la población, sin contar con el subregistro que tiene lugar dentro de la misma institución, ni con el hecho de que no se recurre a las actas de defunción asentadas en los registros civiles. Como consecuencia, las instituciones de salud no tienen la posibilidad de hablar fidedignamente sobre los índices de diabetes en el estado.

Al revisar las actas de defunción del municipio de Tenejapa hemos encontrado que, de constituir la séptima causa de muerte en 2008 con cuatro decesos, pasó a ser la primera en 2010 con 18 casos, sosteniéndose en dicho lugar hasta el presente año 2012. 
Lo anterior contrasta con la cantidad de diabéticos con los que el investigador y su equipo se toparon en las comunidades donde se realizó el trabajo, la mayoría de ellos alejados de la atención de las instituciones de salud por opción propia como resultado del desencanto que han generado décadas de ineficiencia. La mayor parte de los diabéticos no utiliza dichos servicios porque considera que ahí no se les controlará su padecimiento. Los que recurren a la biomedicina lo hacen acudiendo, según el caso, al Hospital de las Culturas en San Cristóbal, a la Clínica de Campo de IMSS-Oportunidades, o a la clínica denominada Esquipulas. Esta última se trata de una clínica de atención financiada por empresarios de Nuevo Léon, y operada por médicos titulados y pasantes en servicio social provenientes de ese estado norteño, la cual se ha ganado el respeto de la población por su buena atención y por el acceso que proporciona a los tratamientos. Otras personas, las que cuentan con mayores recursos, acuden a la atención privada. Por ejemplo, de 30 diabéticos entrevistados en la cabecera municipal de Tenejapa, solamente dos asistieron a centros de salud. Los demás, en el mejor de los casos buscaron otras alternativas, y en otros simplemente no hicieron nada al respecto.

\section{Homicidio-dulce-suicidio}

No es posible afirmar que todo diabético maya de las localidades que nos ocupan lo sea debido solamente al consumo de refrescos. Sin embargo, la mayoría ha incurrido en sus vidas a su consumo cotidiano, predominantemente de Coca-Cola.

En entrevistas realizadas en las cabeceras municipales de Chamula, Tenejapa y San Cristóbal de Las Casas, se detectó que en Chamula es más frecuente la relación refrescos-diabetes, seguido por Tenejapa y finalmente por San Cristóbal. Lo anterior constituye tan sólo una tendencia que permite formular hipótesis a confirmar o descartar.
Un factor de enorme importancia en el consumo de refresco es la vida socio-religiosa que tiene lugar en Chamula y Tenejapa, pero principalmente en el primero de estos dos municipios. Cuando a alguien se le designa para una mayordomía, se da por sentado que eso implica el involucramiento de la familia y de la mayor parte de las personas que forman parte de su red. Todo evento que esté relacionado con dicho cargo implica ritualidad y comensalidad, pero sobre todo la ingestión continua de bebidas, entre las que predomina la Coca-Cola. Si bien también se ofrece pox - aguardiente de caña-y cerveza, predomina el refresco.

En la percepción de los diabéticos y sus familiares en relación con la génesis de la enfermedad, hay coincidencias en atribuir su origen a varios años de consumo incontrolado de refresco, en tanto que su desencadenamiento se liga a algún evento crítico que haya implicado considerable sufrimiento.

Con eso me dio la enfermedad, es que en ese día cuando tenía coraje me dieron de tomar Coca-Cola y al siguiente día amanecí mal, sentía que se secaba mi boca y de repente me empezó a dar mucha sed, tenía cansancio, de ahí compramos vitaminas pero no me hizo nada (Sebastiana 2).

Estaba yo llegando a trabajar en Cancún, allí me empezó, porque allí la temperatura es alta, es donde me empezó la sed, a tomar mucha Coca y me daba mucha hambre. Pero entonces, por fin vine a dar servicio aquí en mi comunidad como comité de educación, acá en esta escuela es donde también aguanté mucha hambre y empecé a consumir refrescos de Coca embotelladas pues también un poquito de coraje de ahí es donde empezó mi enfermedad (Carmelino).

¿Sabes cómo me empezó?, es que yo tomaba refresco antes de comer. Es que estaba trabajando en una balconería, hasta en la comunidad de Yitic, ahí fui a trabajar, en una balconería, después como a los ocho meses o siete, empecé a tomar mucha Coca, es que no 
había quién me daba la comida luego, el refresco me quitaba el hambre, es por eso que ahí me empezó, si así me empezó, y no lo sabía yo (Salvador).

Pues una parte porque aquí en la comunidad, la cólera que hay que tomar mucha Coca-Cola dicen (se ríe) dicen pues, las enfermeras, pues, que hay que tomar dos tres Coca Colas diario para hidratarse, fijjate, está cabrón je, je, je (Juan).

Pues anteriormente mi mamá estaba buena. Como mis padres eran encargados de cada paxion para que le aconsejaran a los paxionetik; ella comía bien y subió un poquito de peso, llegó a 75 kilos, estaba tranquila después un tiempo se fueron en Chamula, y es donde le daba mucha sed, tomaba 2 o 4 refrescos diarios y después regresaron en la casa, pero siguió igual con la sed, pasó días hasta que ella se preocupó de su salud porque estaba orinando cada ratito pero como nosotros no sabíamos si era la enfermedad diabética (María X).

Suelen transcurrir varios años antes de que quien padece diabetes se percate de que está enfermo. En esos años la enfermedad avanza inexorablemente: la gente orina descontroladamente, después de ser obesa adelgaza sin razón aparente, disminuye sensiblemente su fuerza y se cansa con rapidez, y disminuye su agudeza visual. Estos síntomas vienen acompañados de un deseo incontrolable por comer e ingerir alimentos y bebidas muy azucarados; aunemos a ello la gran disponibilidad que hay de Coca-Cola a un precio bastante accesible para los pobres a pocos pasos de cualquier vivienda.

Así no más, así no más y me agarró así no más, solamente quiero tomar refresco todos los días, entonces tomé dos [refrescos], ni con eso se calmó mi corazón o la sed, a los cuatro refrescos se calmó mi corazón... (María 2).

Sí mucho tiempo, creo que llevó quince años, como antes teníamos cargos, vendemos el refresco y cualquier persona nos invita a tomar el refresco y es lo que tomamos. Allí empezó. Teníamos cargos religiosos entonces, cualquier persona te invita a tomar el refresco. Entonces, durante quince años tomaba mucho refresco, pero era como uno o dos al día, pero entonces empezó a dar más sed y empezó a tomar cuatro refrescos, y en la noche ya no podía parar al baño, entonces se dio cuenta que ya no es normal... (María 2).

Una vez los operadores médicos informan a los pacientes del diagnóstico, les ofrecen indicaciones en la mayoría de los casos incompletas. Se les informa de que se trata de una enfermedad incurable que sólo puede controlarse y de que gradualmente, sobre todo si la persona no se cuida, desemboca en severas complicaciones y eventualmente en la muerte. A ello le sigue la determinante orden de modificar los hábitos de vida, principalmente la dieta, también sin mayor explicación: nada de refresco, dos o tres tortillas máximo, no comer dulces, no comer grasas, poca carne...

¿Dejar la tortilla?, ¿no tomar Coca?, ¿y entonces...? La mayor parte de los diabéticos de nuestro universo de trabajo no están dispuestos a someterse sin mayor explicación a una dieta en la que tienen que renunciar a los elementos dietéticos más importantes dentro de la cultura culinaria en la que nacieron y crecieron, y a la que tienen fuerte adhesión, incluida la ya incorporada Coca-Cola.

¿Cómo le ayudó? Le prohibieron tomar refresco, nosotros no le damos, pero es que ella lo toma a escondidas, si no le damos aquí en la tienda se sale y lo compra en otra tienda y lo consume a escondidas, todo lo que es dulce, lo consume el café; toma refresco, consume todo lo que es dulce, hasta paletas, no nos hace caso, lo toma a escondidas y todo lo que es dulce es lo que quiere ella, el doctor me dijo que hay que cuidar su alimentación] (Verónica). 
Al asomarme a la vivienda, me topo con María, quien sentada en una silla, sostiene una Coca-Cola en la mano. Al verme se ríe y coloca el refresco sobre una mesa para hablar conmigo. Ella está atendiendo a una joven mujer que la visita, que también está tomando refresco (María G.).

No todo, no lo puedo hacer, lo que no lo puedo dejar de tomar es el refresco, sólo que ya no se compara como antes tomaba, ya tomo poco, así como el café tomo muy poquito, pero cuando quiero tomar no obedezco (Magdalena).

Encontramos también testimonios de otros tipos, como que, aunque tengan el "azúcar" elevado, se sienten bien, o que al normalizarse la glucemia con los medicamentos proporcionados en la clínica se sienten débiles. Por este motivo abandonan sus tratamientos, lo cual sucede con inusitada frecuencia, dato que fue reforzado a partir de las experiencias de la médica adscrita a la UMR de Tenejapa (entrevista 22 de febrero de 2012). Ella informó que retoman los hábitos alimenticios de toda su vida reduciendo un poco la ingestión de refresco y de tortilla. Asimismo, otras personas, al normalizarse la glucemia, abandonan sus tratamientos, sean de medicamentos o de plantas medicinales, porque alguien les dice que ya están curados; eso hasta que se presentan las complicaciones.

Si se cuecen las dos clases y lo tomo igual, me dijeron que no es necesario la dieta, que puedo tomar el refresco, hasta puedes comer chile, me dijeron. No parece (que sube mucho el azúcar), tampoco tomo así exageradamente, porque también dejé de tomarlo, apenas la mitad tomo, ya no acabo una botella (Rosa).

Pareciera que el panorama que se presenta pudiera generalizarse entre nuestros colaboradores, pero no es así. También nos hemos topado con enfermos y sus familiares informados y conscientes de lo dañinos que son los alimentos chatarra, quienes además con suma inquietud se preguntan: "iPor qué la tortilla también sube el azúcar?", "ipor qué los médicos la evitan —les indican que la retiren de la dieta-?"

Hemos notado que, en general, cuando hay disponibilidad por parte del médico o del antropólogo, la gente muestra interés por entender los pormenores de la enfermedad y de las medidas a tomar $y$, una vez que han asimilado las explicaciones, algunos hacen esfuerzos por lograr el control de su glucemia.

No, ese no lo sube, porque siempre estoy preguntando cómo siente ese de comida. Cómo siente, si puede comer carne, no lo hace nada. Cuando lo hace, entonces no lo come. Ansí todo estoy preguntando, sea verdura, sea carne y carne cocida. Si lo hace entonces ya no lo come. Si por ejemplo, del caldo, lo mueve ese el azúcar, mejor ya no lo come (María S.).

\section{Comentario final}

El concepto de vulnerabilidad engloba las condiciones de subalternidad-subordinación y exclusión a las que están sometidos los pueblos originarios, lo que, entre otras cosas, coadyuva a la persistencia e incorporación de prácticas que dañan la salud. Dentro de estos factores destacan, en función del tema que nos ha ocupado, los rezagos en educación y salud, que derivan en la sujeción de la población a grupos caciquiles que controlan las instancias de organización socio-religiosa y han logrado, en contubernio con las empresas que les han cedido concesiones, como para el caso son Coca-Cola y Pepsi, imponer prácticas entre los habitantes que les benefician económicamente, independientemente de los costos socioculturales y de salud que impliquen.

La relación vertical que marca la subalternidadsubordinación a favor de los actores estructuralmente ubicados en el grupo dominante, la cual se retroalimenta a través de diferentes mecanismos, constituye en el 
marco de la vulnerabilidad la condición que facilita la imposición de criterios para el consumo, la cual se fortalece con estrategias de mercado como la publicidad excesiva, el abaratamiento de los productos y su ubicación entre los que nada tienen como símbolo de estatus.

Elproducto, en este caso la Coca-Cola, no encuentra sus espacios de venta por sí solo. En los procesos correspondientes inciden, además de los arriba señalados, diferentes actores a través de los cuales se condicionan representaciones y prácticas. Para el caso, por ejemplo, se encuentran los grupos religiosos protestantes que, como agentes del capitalismo, buscan la integración de sus feligreses a la visión capitalista del mundo. Para este fin, el discurso proselitista busca erradicar, en principio, las prácticas religiosas centradas en la combinación de elementos prehispánicos y católicos mediante un discurso satanizador dirigido a dichas representaciones y sus prácticas. Entre los grupos religiosos víctimas de tal escalada, esta situación ha ocasionado cambios significativos en los núcleos fundamentales de sus concepciones religiosas, en aras de lograr consensos que permitan la persistencia de sus formas religiosas sin entrar en conflicto con la sociedad dominante.

Entre los elementos más ferozmente atacados por los protestantes está el consumo de aguardiente, que a la larga ha sido remplazado en la ritualidad y en el acontecer lúdico y cotidiano por productos como la Coca-Cola, productos no sólo aceptados por estas Iglesias, sino indirectamente promovidos desde su seno, y que en el rejuego económico benefician tanto a la empresa embotelladora como a los grupos de poder que detentan las concesiones en los diferentes municipios.

A lo antedicho se aúnan la mercadotecnia empresarial, el discurso en salud que favorece, por ejemplo, el uso del refresco como medio para la rehidratación ante la diarrea y el vómito, o el control de los productos por parte de los grupos de poder de los municipios.
En este ámbito, la vulnerabilidad remite entonces a la fragilidad del receptor que, frente a la exclusión en educación y las presiones sociales y religiosas, cede con facilidad ante lo espectacular del ofrecimiento publicitario, el cual se ve retroalimentado con el efecto de llenado gástrico y energetizador del refresco, así como con la obtención de estatus y respeto comunitario al ofrecer estos productos bien situados en los eventos sociales y religiosos que encabezan, y que han dado pie a la obesidad y la diabetes, dos epidemias estrechamente ligadas cuyas complicaciones generan la más elevada mortalidad en México.

Uno de los problemas centrales en torno a dichas enfermedades y a la importancia que tiene el consumo principalmente de refresco, pero también de fritos, dulces y galletas, es la falta de iniciativas por parte de las instancias a las que corresponde paliar los fenómenos referidos, es decir, las instancias del sector salud.

En este sentido, por un lado es alarmante y por otro desolador que dentro de los objetivos del milenio relacionados con la salud no aparezcan ni la obesidad ni la diabetes como prioridades a atender (NCD Alliance, 2012). Por otro lado, en el Plan Nacional de Salud 2006 2012 se plantea realizar acciones para atender estos problemas, esencialmente de orden preventivo, las cuales no son instrumentadas en los servicios de salud de primer nivel presentes en las comunidades, debido, como ya se señaló, a que en estos se opera curando enfermedades y atendiendo la demanda.

Sin embargo, no todo está perdido. Hemos registrado, al menos entre quienes han colaborado en el proyecto, familias que han tomado medidas que coadyuvan a evitar y limitar la aparición y daño por diabetes, hecho que se incorpora a las redes de comunicación establecidas en dichos grupos. Para ello se apoyan en lo que gradualmente van conociendo de las particularidades de la enfermedad y sus causalidades tangibles, todo ello ligado, por supuesto, a las experiencias y sintomatología de los afectados. Expresan su preocupación por el consumo excesivo de 
refresco y lo difícil que será contener y controlar dicho consumo a nivel poblacional, pero al menos algunos ya toman medidas en el seño familiar:

[...] puro refresco, pero está muy dulce, ipor qué?, porque puro azúcar, ahí es donde nos pega, se vuelve dulce la sangre. Me platicó un mi amigo que ya no se puede tomar mucho refresco, hay que evitarlo ese, para eso hay agua; se puede tomar café sin azúcar, se puede tomar un te sin azúcar, o si no, se puede tomar agua simple, es mucho mejor (Lorenzo, audio, 21 de marzo de 2012).

\section{Agradecimientos}

El proyecto gracias al cual ha sido posible este trabajo recibe financiamiento del Fondo de Investigación Básica SEP-CONACYT, clave CB-2008-01-99859.

\section{Bibliografía}

Blanding, Michael (2010), The Coke Machine. The dirty truth behind the world's favorite soft drink, Nueva York: AveryPenguin Group.

EFE (2012), “Coca Cola invertirá 1.000 millones de dólares en México en 2012", en RPP Noticias, 28 de enero. 〈http://www.rpp.com.pe/2012-01-28-coca-colainvertira-1-000-millones-de-dolares-en-mexico-en2012-noticia_445848.html> [2 de febrero de 2012].

Encuesta Nacional de Salud y Nutrición 2006 (2007), Resultados por entidad federativa, Chiapas. México: Instituto Nacional de Salud Pública.

Farmer, Paul (2001), Infections and Inequalities. The modern plagues, Estados Unidos: University of California Press.

Favre, Henri (1973), Cambio y continuidad entre los mayas de México, México: Siglo XXI.

Guthrie, Amy (2012), "Coca-Cola invertirá US\$5.000 millones en México por cinco años”, en The Wall Street
Journal Americas, 30 de enero. 〈http://online.wsj.com/ article/SB10001424052970204740904577193200450 010384.html> [2 de febrero de 2012].

INEGI(2010), Cuéntame...deMéxico, México:INEGI. ‘http:// cuentame.inegi.org.mx//monografias/informacion/ chis/poblacion/default.aspx?tema=me\&e=07 $\quad[30 \mathrm{de}$ julio de 2012].

Jordan, Laura K. (2008), El problema de la responsabilidad social corporativa: La empresa Coca-Cola en Los Altos de Chiapas, (tesis de maestría), Centro de Estudios Superiores en Antropología Social-Sureste, San Cristóbal de Las Casas, Chiapas.

Menéndez, Eduardo L. (2002), La parte negada de la cultura. Relativismo, diferencias y racismo, España: Ediciones Bellaterra.

NCD Alliance (2012), "World TB Day. The Union and the World Diabetes Foundation call for greater awareness of TB-diabetes co-epidemi". 〈http://ncdalliance.org/ news/world-tb-day-union-and-world-diabetesfoundation-call-greater-awareness-tb-diabetes-coepidemic [10 de noviembre de 2012].

OCDE (2010), La obesidad y la economía de la prevención. 〈http://www.oecd.org/centrodemexico/medios/ obesidadylaeconomiasdelaprevencion.htm $>[30 \mathrm{de}$ julio de 2012].

Page Pliego, Jaime (2005), El mandato de los dioses: medicina entre los tzotziles de Chamula y Chenalhó, Chiapas, México: Programa de Investigaciones Multidisciplinarias sobre Mesoamérica y el Sureste-Instituto de Investigaciones Antropológicas de la Universidad Nacional Autónoma de México.

Pérez Lizaur, Ana Bertha, Berenice Palacio González y Ana Laura Castro Becerra (2012), Sistema mexicano de alimentos equivalentes, México: Instituto Nacional de Nutrición Salvador Zubirán.

Robledo Hernández, Gabriela Patricia (1987), Disidencia y religión: los expulsados de San Juan Chamula, (tesis de licenciatura), Escuela Nacional de Antropología e Historia, México. 
Yáñez Moreno, Pedro (2012), He hipi cöhimoqueepe hac hocoaa ha, izooh sah pacta teeh? Sé que estoy enfermo, ¿Y qué hacer? La diabetes mellitus y sus significados entre los comcaac de Socaaix (Punta Chueca), Sonora, (tesis de maestría), Colegio de Sonora, Sonora. 
Foto 1. Planta Coca Cola-FEMSA en San Cristóbal de Las Casas.

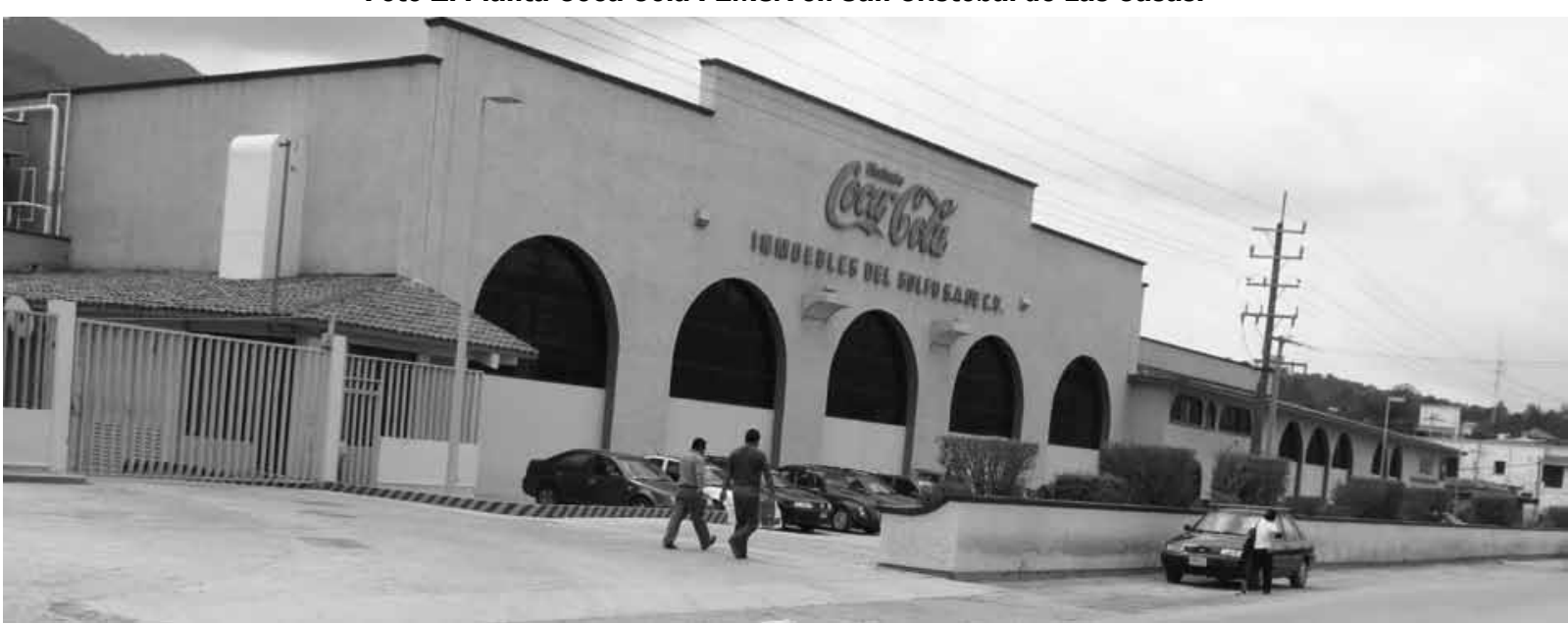

Fuente: Jaime Page.

Foto 2. Planta Coca-Cola FEMSA de San Cristóbal de Las Casas. Al fondo, el cerro del Huitepec.

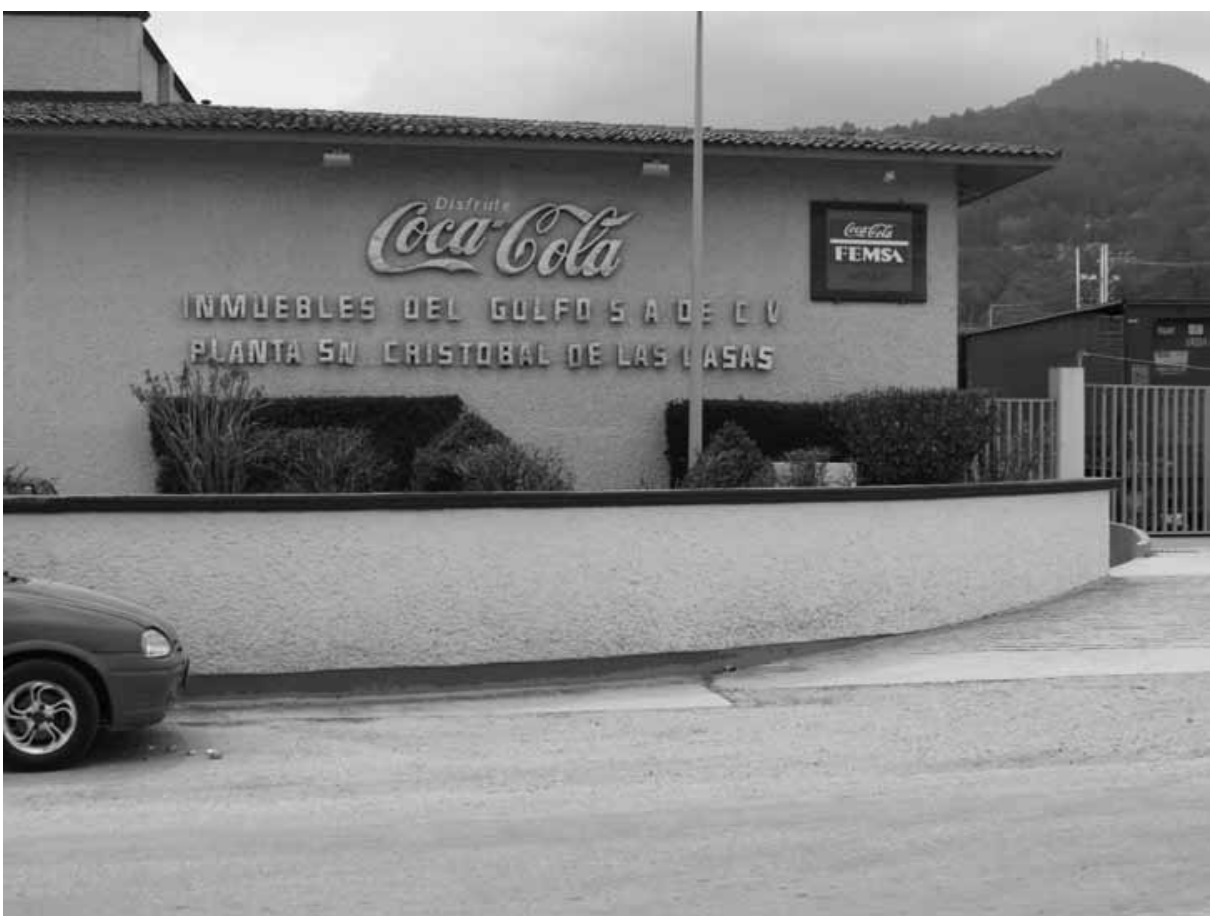

Fuente: Jaime Page. 
Fotos 3 y 4. Anuncios de bienvenida.
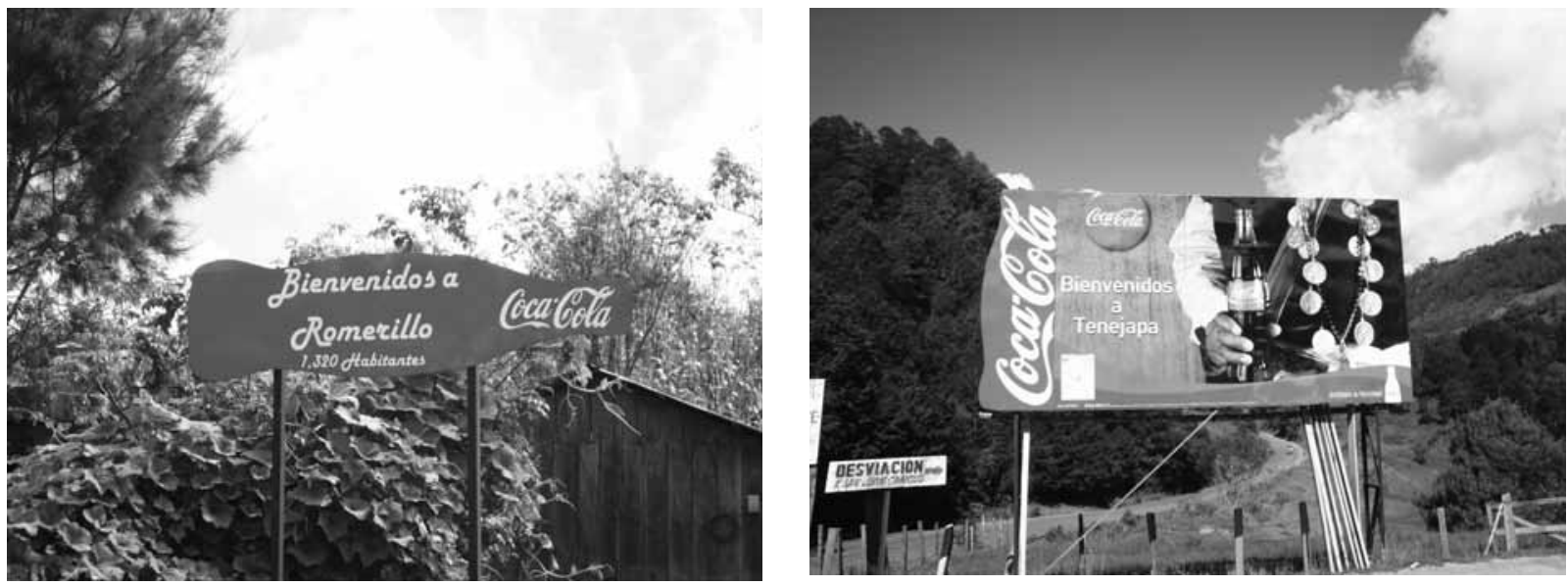

Fuente: Jaime Page.

Foto 5. Depósito de Coca-Cola en Chalchihuitán, Chiapas.

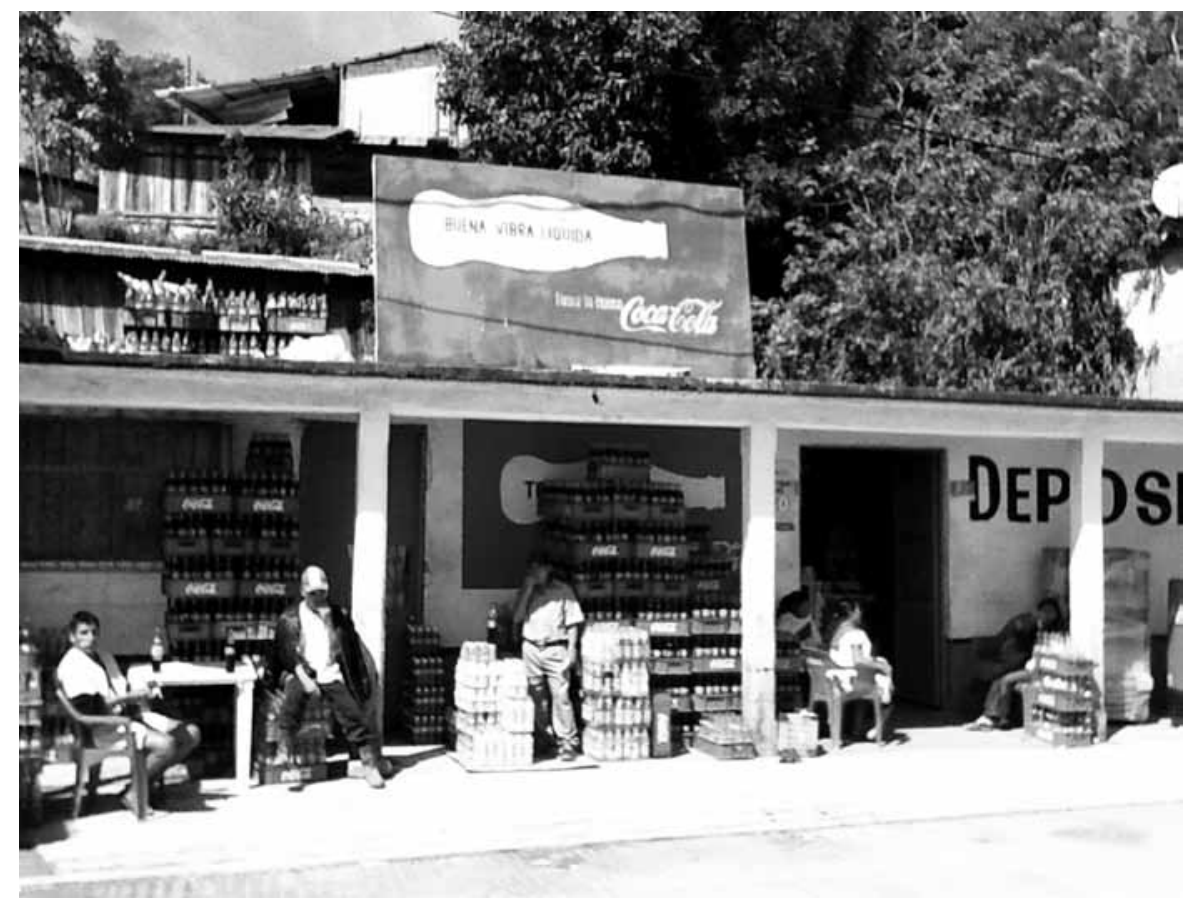

Fuente: Jaime Page. 